\title{
Real-Time Operation Management of CHP System in Manufacturing Industry
}

\author{
Pouya Ghadimi ${ }^{1}$, Sami Kara ${ }^{1} \&$ Bernard Kornfeld ${ }^{1}$ \\ ${ }^{1}$ School of Mechanical and Manufacturing Engineering, Sustainable Manufacturing and Life Cycle Engineering \\ Research Group, The University of New South Wales, Sydney, Australia \\ Correspondence: Pouya Ghadimi, School of Mechanical and Manufacturing Engineering, The University of New \\ South Wales, Sydney, 2052, Australia. Tel: 61-449-877-892. E-mail: pouya.ghadimi@gmail.com
}

Received: Ooctober 12, 2014

Accepted: October 23, $2014 \quad$ Online Published: January 10, 2015

doi:10.5539/mas.v9n2p158

URL: http://dx.doi.org/10.5539/mas.v9n2p158

\begin{abstract}
In this paper an integrated simulation and optimization approach for real-time operation management of on-site energy systems within manufacturing facilities is proposed and evaluated. Expected outcomes of on-site energy systems are highly dependent not only on system sizing but are also affected by system utilisation. The latter is dependent on the choice of operational management strategy for integrated components. Although fixed operational strategies can guarantee reliable performance and enhance the outcomes of individual components, system effectiveness can drop severely at the utilisation stage in the presence of multiple energy supply options and operating environment dynamics. There is, therefore, a need for autonomous operation management systems that can compensate for the existing shortcomings of fixed operational strategies. Real-time optimization of controllable options has shown potential for continuous and optimal decision-making. However the lack of a reliable, optimal and practical solution has been identified.

In this paper we present, an exemplar model with real-time application, which not only covers the mechanical integrity of the components, but also address the specific requirements for real-time application. In addition, a simulation-based methodology for real-time optimization is described. The proposed autonomous management scheme would continuously guarantee system reliability, unit commitment, and optimal operational objectives. Finally the proposed approach is simulated and evaluated for the case study of an existing manufacturing plant. The results illustrate the potential for improvement as well as applicability compared to existing operational management approaches.
\end{abstract}

Keywords: on-site energy system, combined heat and power, autonomous operation management, real-time optimization

\section{Introduction}

On-site energy generation may deliver enormous economic and environmental advantages for manufacturing industries (Meckler \& Hyman, 2009; Turner, 2004). This can be achieved by integration of potential energy generators such as combined heat and power (CHP) systems into manufacturing plants (Ghadimi, Kara, \& Kornfeld, 2013). This approach not only has the potential to reduce the operational cost and environmental impact of manufacturing plants, but can also contribute to a $30-40 \%$ increase in energy efficiency (Meckler \& Hyman, 2009).

Optimal sizing at the system configuration stage plays a significant role in achieving expected outcomes (Ghadimi et al., 2013; Liu, Shi, \& Fang, 2013). However the choice of operational strategy for integrated generators can severely impact the system effectiveness at the operation stage (Ghadimi, Kara, \& Kornfeld, 2014b; Hueffed \& Mago, 2010; Oh, Lee, Jung, \& Kwak, 2007). This is mainly restricted to fixed operational strategies (electrical load following (ELF) or thermal load following (TLF)) that seek to align an individual generator's operation to instantaneous applied loads. In this case further enhancement is achievable through optimal operation plans (Andreassi, Ciminelli, Feola, \& Ubertini, 2009).

However these approaches do not take into account the time-varying nature and uncertainty associated at the operation stage of on-site energy systems (Flores, Shaffer, \& Brouwer, 2014; Ghadimi et al., 2014b; Mitra, Grossmann, Pinto, \& Arora, 2012; Gamou, Yokoyama, \& Ito, 2002). In this case operation of the energy systems 
can be subject to several time-dependent factors such as dynamic energy demand of manufacturing plants, real-time energy pricing, uncertain availability of the generators and their potential intermittency (Carvalho, Romero, Shields, \& Millar, 2014; Firestone \& Berkeley, 2007; Lozano, Carvalho, \& Serra, 2011). For example, figure 1 illustrates the dynamics of power and heat demand of the studied manufacturing plant over two weeks caused by varying production plans. Consequently these operational strategies result in static optimization and offline solutions where their application is restricted as planning tools (Baños et al., 2011; Gamou et al., 2002; Hiremath, Shikha, \& Ravindranath, 2007).
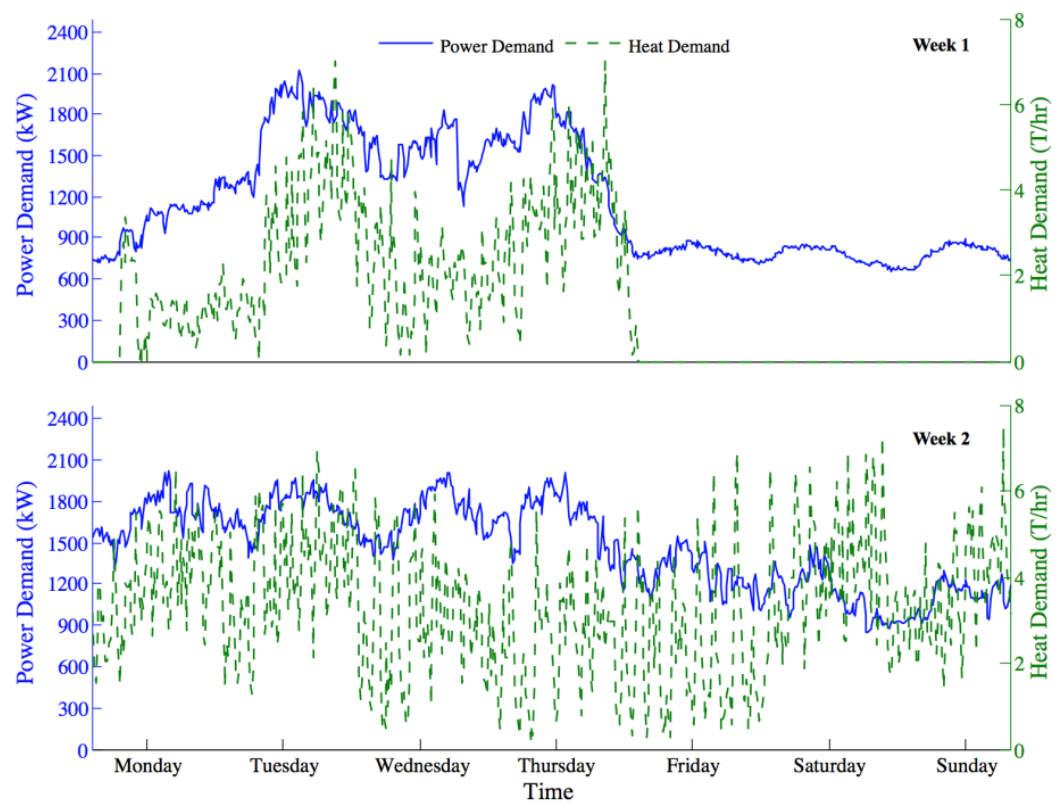

Figure 1. Power and heat demand fluctuation of the studied manufacturing plant over two weeks

Altogether the operational management of on-site energy systems is a dynamic problem that requires a real-time solution (Bonvin, Srinivasan, \& Ruppen, 2002; Vallianou \& Frangopoulos, 2012). In this case the model predictive control (MPC) method is used to assure optimal operation of CHP systems in a continuous manner (Cho, Luck, \& Chamra, 2010; Vallianou \& Frangopoulos, 2012; Yun, Cho, Luck, \& Mago, 2011). These studies form a closed loop approach and have demonstrated around $18 \%$ cost savings. Additionally the multi-agent approach has been studied to achieve autonomous and continuous operation management of energy systems (Kim, Lim, \& Kinoshita, 2012; Logenthiran, Srinivasan, Khambadkone, \& Aung, 2012; Meiqin, Wei, \& Chang, 2011; Dimeas \& Hatziargyriou, 2005). Compared with MPC approach this can take into account interactions among different system components as agents through communication channels. However unlike MPC approaches, they cannot guarantee optimal results since they are based on operational rules and bidding strategy where system forecasts are not taken into account (Kim et al., 2012; Kim, Kinoshita, \& Shin, 2010; Smitha \& Chacko, 2013).

Despite the suggested advantages of real-time operation management, the lack of reliable, optimal and practical solution that can be applied continuously is a major shortcoming that is explained at the Background section of this paper. As a result, the fixed operational strategy is still the dominant operational approach to managing CHP assets within manufacturing plants. Therefore the problem is still an open research topic, which requires the development of comprehensive models and real-time operation management solutions to assure optimal system utilization subject to operating environment dynamics. This should also form the basis for renewable energy supply and storage integration in the future (Wille-Haussmann, Erge, \& Wittwer, 2010).

An integrated simulation and optimization approach is proposed in this paper to address existing shortcomings. The rest of this paper is organized as follows; real-time execution requirements and the limitations of existing modeling approaches are identified in the background section. The methodology section describes details of the proposed solution and underlying developed models. Next, a case study with results generated through the application of the developed methodology is presented. Finally, the conclusion section summaries the potential outcomes and future work that need to be conducted. 


\section{Background}

The first requirement for real-time operation of on-site energy systems is reliability and this requires the application of comprehensive models of the system components to assure unit commitment (Chang, Tsai, Lai, \& Chung, 2004). In this case each generator has different operational states such as purge, initiation and cooling down with specific state based characteristics (fuel consumption rate, ramp up/down rates or efficiency). Moreover associated dynamic transitions such as, block times or minimum up/down times should be integrated accordingly (Ghadimi, Kara, \& Kornfeld, 2014a; Mitra, Sun, \& Grossmann, 2013). As a result two state (on and off) assumption of existing mathematical modelling approaches cannot comprehensively represent system component behavior for continuous and timely applications (Colson, Nehrir, \& Pourmousavi, 2010; Kraning, Chu, Lavaei, \& Boyd, 2012).

In this case, although mode based modelling takes into account operational states, it results in a deterministic model, where associated transitions are only time and condition driven (Mitra et al., 2013). Therefore this type of modelling cannot cover the fundamental requirement of being interactive with regards to system dynamics and interaction between integrated components at the operation stage (Ghadimi et al., 2014a; Kim et al., 2012; Logenthiran et al., 2012).

Furthermore to ensure the reliability of system operation, developed models should be precise enough to represent system behaviors and their associated constraints in real-time, in other words second or even millisecond intervals. In this case, one of the constraints that should be modelled effectively to represent unit commitment is the ramp rate of the generators. Ramp rate represents the capability of the generators in ramping up or down over any two successive time steps. Although existing studies have modelled this constraint over long time intervals (15 minutes to one hour), they do not adequately represent the component behavior in short time intervals (Kraning et al., 2012; Xie \& Ilić, 2009). In fact the long time intervals assumed in the reviewed case studies would permit a generator to ramp down from any instantaneous applied load to the off state and then ramp up back from off mode to minimum applicable load. For instance, a mid-sized reciprocating engine (1 MW) with $6 \frac{\mathrm{kW}}{\mathrm{sec}}$ ramp rate can ramp up or down up to 5 times its rated capacity in the course of 15 minutes $\left(6 \frac{\mathrm{kW}}{\mathrm{sec}} \times 60 \mathrm{sec} \times 15 \mathrm{~min}=5400 \mathrm{~kW}=5.4 \mathrm{MW}\right)$. Consequently ramp rate constraints can be redundant in representing generator behavior in models that assume long time intervals. This is illustrated in figure 2, which shows the power demand fluctuation of the studied manufacturing plant over one day with a resolution of 15 seconds. If this demand were modelled at long time intervals, we might assume that the engine could completely follow the load fluctuations, however in reality the generator is constrained to respond at very short time intervals.

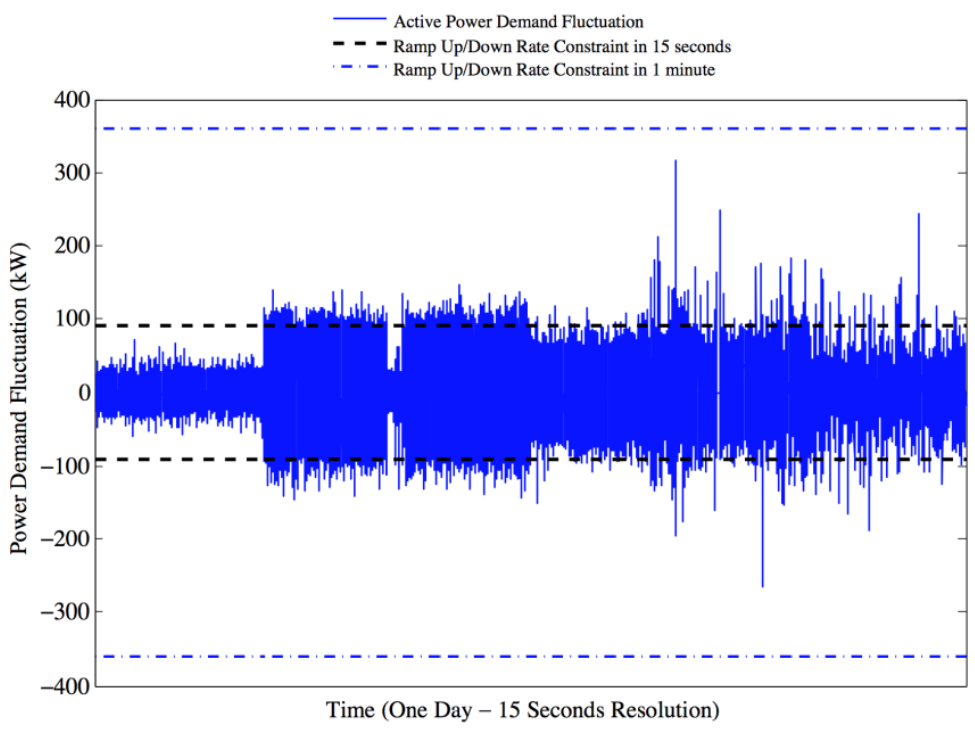

Figure 2. Ramp rate constraint subject to long and short time intervals with regards to power demand fluctuations

In addition, existing ramp rate modelling approaches subject to short time intervals, force generators to permanently keep their initial operating state to prevent constraint violation even though switching to off or on 
mode might be an optimal solution (Carrión \& Arroyo, 2006). To demonstrate this, a generator with a $90 \frac{\mathrm{kW}}{15 \mathrm{sec}}$ ramp rate cannot switch to the off state as it needs to ramp down at least from the minimum applicable load ( 300 $\mathrm{kW}$ ) to $0 \mathrm{~kW}$ in 15 seconds which will violate the $90 \frac{\mathrm{kW}}{15 \mathrm{sec}}$ constraint. As a result, mathematical models that assume long time intervals cannot be used for real-time applications. This therefore necessitates a different modelling approach for real-time applications.

Moreover, to assure system reliability dynamic operational constraints should be integrated into the problem formulation in order to achieve continuous and reliable operation of the on-site energy systems. In fact the problem cannot be considered as a sequence of independent time intervals in which constraints are of a static nature and are generally assumed to be unchanged during system operation. On the contrary, there are time dependent constraints that get changed along the system operation and based on previous decisions (Kim et al., 2010; Yun et al., 2011). For instance, the unavailability of generators due to block time or minimum downtime in addition to accumulated utilization rate and network charges can be considered as dynamic operational constraints. As a result consideration of dynamic constraints requires integration of the system operation history into the problem formulation.

As a second requirement, any proposed solution should continuously generate optimal operational signals for integrated components. This is usually formulated as a deterministic optimization problem whereas there is a possibility that operating environment conditions deviate from what is expected at the operation stage (Mitra et al., 2012; Gamou et al., 2002). Consequently, the operational management scheme should be capable of including not only the current system condition but also potential future dynamics along the rolling control time horizon (CTH) (Bausa \& Tsatsaronis, 1999; Douglass, 2002; Mayhorn et al., 2012; Vallianou \& Frangopoulos, 2012).

Finally the proposed solution needs to be implemented at short time intervals over the rolling CTH, especially if the system consists of renewable energy technologies with intermittent output (Kraning et al., 2012). As a result the proposed solution should be computationally inexpensive. In this case, mathematical formulations that are based on pure optimization methods are subject to serious limitations to be executed in real-time where the model is overloaded subject to presence of several integrated components, operating environment dynamics and coverage of long CTH (Mitra et al., 2013; Marshman, Chmelyk, Sidhu, Gopaluni, \& Dumont, 2010). As a result, although there are studies that use mathematical modelling or scenario-based approaches for optimization, they lose their interpretability and practicality for real-time applications and get limited to optimal operation planning purposes (Salgado \& Pedrero, 2008). Balanced against there are studies that relax the unit commitment problem or convert it into a convex form to be solved in polynomial time (Kraning et al., 2012; Rong, Hakonen, \& Lahdelma, 2009). However this approach requires assumptions that will disregard the reliability requirement at the operation stage.

In summary, to the knowledge of the authors, most of the work in this field is best suited to offline application. Consequently there is need to develop an enhanced operational strategy that can compensate for the aforementioned shortcomings. An integrated simulation and optimization approach that decomposes the problem formulation and can cope with concurrent computation and communication requirements is introduced in this paper. This will fulfill existing quest for a reliable, optimal and practical solution to autonomously manage on-site energy system in real-time, which was the major driver for this study. The following section describes details of the proposed methodology and its underlying modelling approaches.

\section{Methodology}

As stated in the background section, existing operational strategies are incapable to continuously cover the reliability and optimality requirements of the CHP systems in a practical manner. The methodology proposed in this study addresses the aforementioned shortcomings by integrating simulation and optimization methods. The following sections describe the details of this approach and its implementation strategy, which aims to enable the real-time operation management of on-site energy systems.

\subsection{Methodology Overview}

Figure 3 provides an overview of the Real-time Integrated Simulation and Optimization methodology (RISO), which consists of two main modules. The first is the simulation module, in which comprehensive models of energy system components, operation history and forecasts are integrated. The second is the optimization module, which carries out potential scenario generation and selection of the optimal solution. 


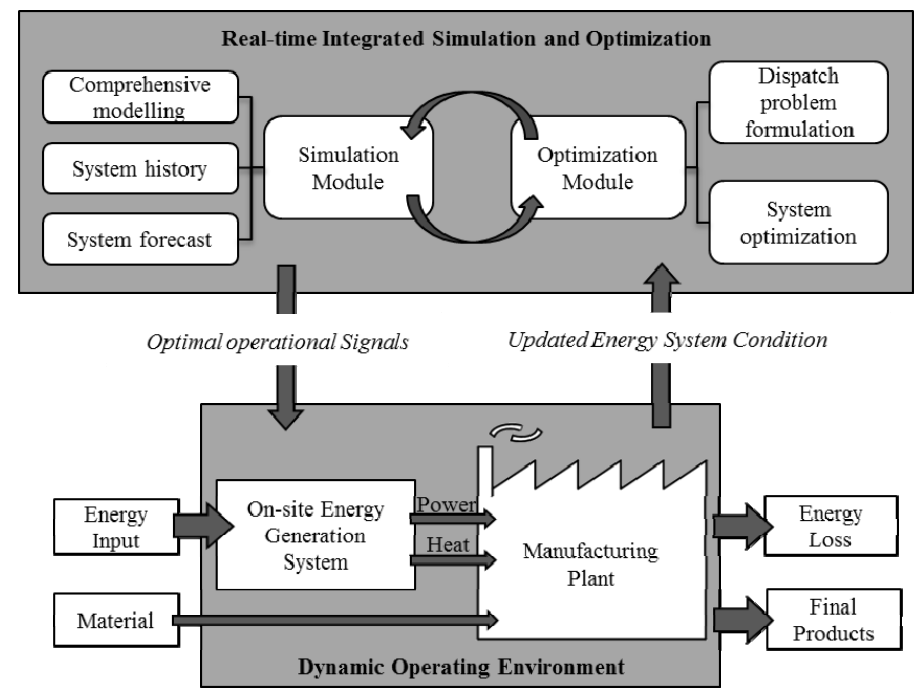

Figure 3. Schematic of Real-time Integrated Simulation and Optimization methodology (RISO)

Optimal operational signals and schedules are generated through interactions between the simulation and optimization modules in polynomial time (Figure 3). Moreover, this can be linked to a dynamic operating environment to receive live system conditions and feedback in order to react to external events. Finally this should be implemented in an iterative manner to cover the rolling CTH and assure continuous management. Details of the individual modules are described as follows.

\subsection{Simulation Module}

The lack of existing modelling approaches to comprehensively represent behaviors of individual component is addressed through the application of state based models. Developed state charts represent transient characteristics of individual states and their interdependencies by different types of transitions (Ghadimi et al., 2014a). This enables a comprehensive coverage of existing non-linearities and state-based constraints.

The inclusion of a message transfer capability as well as dedicated transitions in developed models enables the system to interact with the dynamic operating environment. This is achieved through the integration of two-way message sending and receiving ports that satisfy communication requirements between multiple system components. This addresses the inflexibility of existing modelling approaches, as they are subject to predefined trajectories and are unable to handle operating environment dynamics. Applied models have been developed and validated in previous studies by the authors to represent system behaviors over short time intervals (Ghadimi et al., 2014a).

Finally, the application of a simulation module enables tracking of the system's operational history and the impact of previous decisions to update problem formulation. Dynamic variables, such as utilization rate, number of state switches and remaining unavailability time are defined to address this requirement. Moreover, the simulation module's forward looking capability can take into account the impact of different operational scenarios and the associated system evolution over the CTH. Consequently any decision will be made with respect to the past decisions as well as a forecast of future system condition. Altogether the aforementioned advantages should guarantee mechanical integrity and reliability of models along with a timely representation of real systems.

\subsection{Optimization Module}

The objective of this study is the real-time economic dispatch of energy system along with a unit commitment guarantee. Firstly the optimization module incorporates high-level energy balance equations to assure consistent energy supply (Ghadimi et al., 2014b). This module also addresses the real-time modeling requirement by examining short time steps. Finally the module generates optimal scenarios based on defined objectives and selects the optimal solution from a generated search space.

\subsection{Methodology Logic}

The following flowchart illustrates the logic behind the applied methodology as a closed loop, which enables an 
iterative implementation of the proposed methodology.

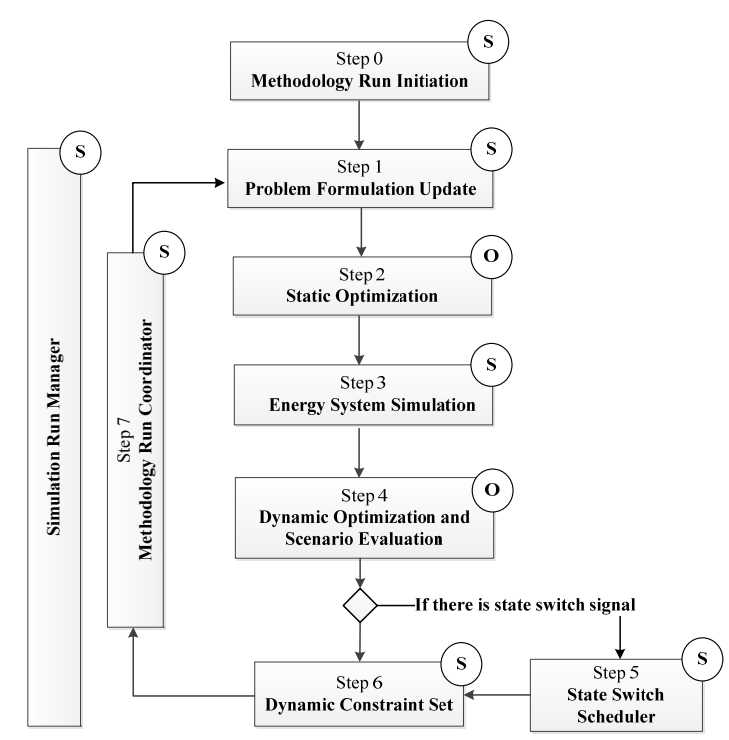

Figure 4. Interaction logic between simulation and optimization modules

In this case each step is executed either in the simulation $(\mathrm{S})$ or optimization $(\mathrm{O})$ module, which are linked to each other. The following sections will provide greater insight into each step.

\subsubsection{Methodology Run Initiation}

The methodology is initiated by checking all underlying assumptions and constraints based on user-defined parameters. As a result, the execution of this step guarantees correct model construction before methodology run.

\subsubsection{Problem Formulation Update}

As the first step of the closed loop methodology, dynamic operational constraints are updated based on the system's operational history to reformulate the economic dispatch problem accordingly. Moreover, this step can be linked to the forecast system, in which potential energy demand of the next CTH is simulated (Herrmann, Thiede, Kara, \& Hesselbach, n.d.; Müller, Stock, \& Schillig, 2014). This should be achieved by taking into account a set of updated production schedules, which determines updated energy demand profiles. The latter would enable concurrent integration of the production plans with associated energy demand and supply.

\subsubsection{Static Optimization}

At this step, static optimization is carried out to generate optimum dispatch solutions for individual time steps along the CTH, subject to unit commitment constraints (Ghadimi et al., 2014b). Moreover, ramp up/down rate constraints between two successive time steps are taken into account at this step. The model has been modified to overcome the inability of existing approaches to comprehensively represent this constraint for short time intervals (Arroyo \& Conejo, 2004; Carpaneto, Cavallero, Freschi, \& Repetto, 2006; Dillon, Edwin, Kochs, \& Taud, 1978; Lee, Lemonidis, \& Liu, 1994). Hence three binary variables and two groups of ramp rates are introduced. These consist of an operation indicator variable (V), in which 1 indicates that optimal generator operation is achieved when it is at on mode and 0 is set when it is optimal to be off. The two other binary variables are state switch indicators $(\mathrm{Y})$, which indicate an off to on state switch (start-up) and $\mathrm{Z}$, which indicates an on to off state switch (shutdown). In addition, specific ramp rates $\left(R R_{u}\right.$ and $\left.R R_{d}\right)$ are introduced alongside existing ramp rate constraints $\left(R_{u}\right.$ and $\left.R_{d}\right)$ to enable a state switch to off or on mode from any applied load at short time intervals (Figure 5). 


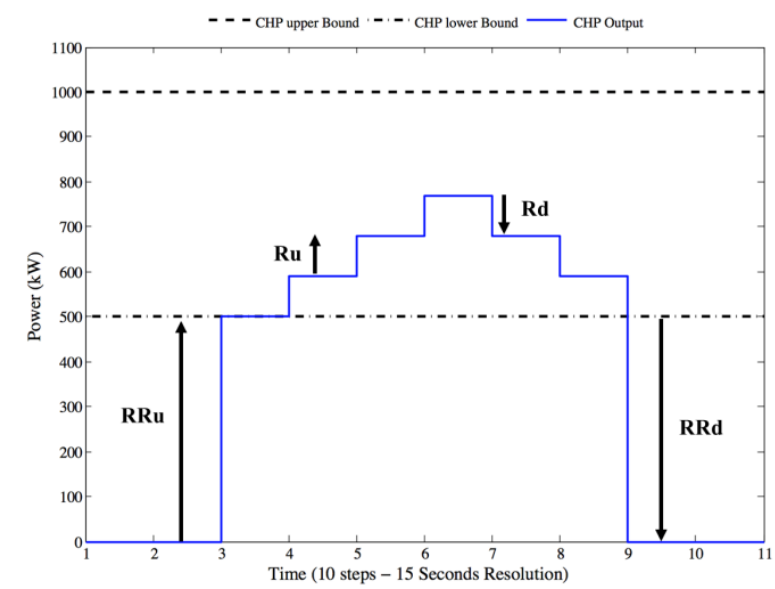

\begin{tabular}{|c|c|c|c|c|c|c|c|c|c|c|}
\hline Time Step & 1 & 2 & 3 & 4 & 5 & 6 & 7 & 8 & 9 & 10 \\
\hline $\mathrm{Vi}$ & 0 & 0 & 1 & 1 & 1 & 1 & 1 & 1 & 0 & 0 \\
\hline $\mathrm{Yi}$ & 0 & 0 & 1 & 0 & 0 & 0 & 0 & 0 & 0 & 0 \\
\hline $\mathrm{Zi}$ & 0 & 0 & 0 & 0 & 0 & 0 & 0 & 0 & 1 & 0 \\
\hline $\mathrm{Ri}$ & $\mathrm{R}$ & $\mathrm{R}$ & $\mathrm{RR}$ & $\mathrm{R}$ & $\mathrm{R}$ & $\mathrm{R}$ & $\mathrm{R}$ & $\mathrm{R}$ & $\mathrm{RR}$ & $\mathrm{R}$ \\
\hline
\end{tabular}

Figure 5. Short-time interval modelling of ramp rate constraint and associated variables

The following equations describe how the model represents the aforementioned constraints:

$$
\begin{gathered}
Y_{i}-Z_{i}=V_{i}-V_{i-1} \\
Y_{i}+Z_{i} \leq 1 \\
E_{d_{i}} \leq E_{G r i d_{i}}+P_{C H P} \cdot t \cdot L_{C H P_{i}} \\
L_{C H P_{i}}-\left(\frac{E_{p}+R_{u} \cdot t}{P_{C H P}}\right) \cdot V_{i}-R R_{u} \cdot t \cdot Y_{i} \leq 0 \\
-L_{C H P_{i}}+\left(\frac{E_{p}-R_{d} \cdot t}{P_{C H P}}\right) \cdot V_{i}-R R_{d} \cdot t \cdot Y_{i} \leq 0 \\
L_{C H P_{i}}-U_{b} \cdot V_{i} \leq 0 \\
-L_{C H P_{i}}+L_{b} \cdot V_{i} \leq 0
\end{gathered}
$$

In which $E_{d_{i}}, E_{G r i d_{i}}$ and $L_{\mathrm{CHP}_{i}}$ are variables that represent energy demand, purchased electricity from the grid and applied load to the CHP system at time step i with t duration. $E_{p}$ indicates previous step's (i-1) applied load on the generator. Moreover $P_{C H P}$ is rated capacity of the CHP system with defined upper bound $\left(U_{b}\right)$ and lower bound $\left(L_{b}\right)$ operational constraints. This optimization problem results in a mixed integer linear program (MILP) that is solved by branch and cut algorithms and can be expanded to cover multiple energy supply options (Currie \& Wilson, 2012).

Figure 6 illustrates information flows between the next two steps of the proposed methodology, which are linked to this step and result in optimal operational signals. At the current step, optimal state switch signals ( $\mathrm{Y}$ and $\mathrm{Z})$ are generated for individual time steps along the CTH regardless of interdependencies between time step decisions. Following this, the scenario matrix (S) is developed, which covers all the potential combinations of generated signals for the integrated components. 


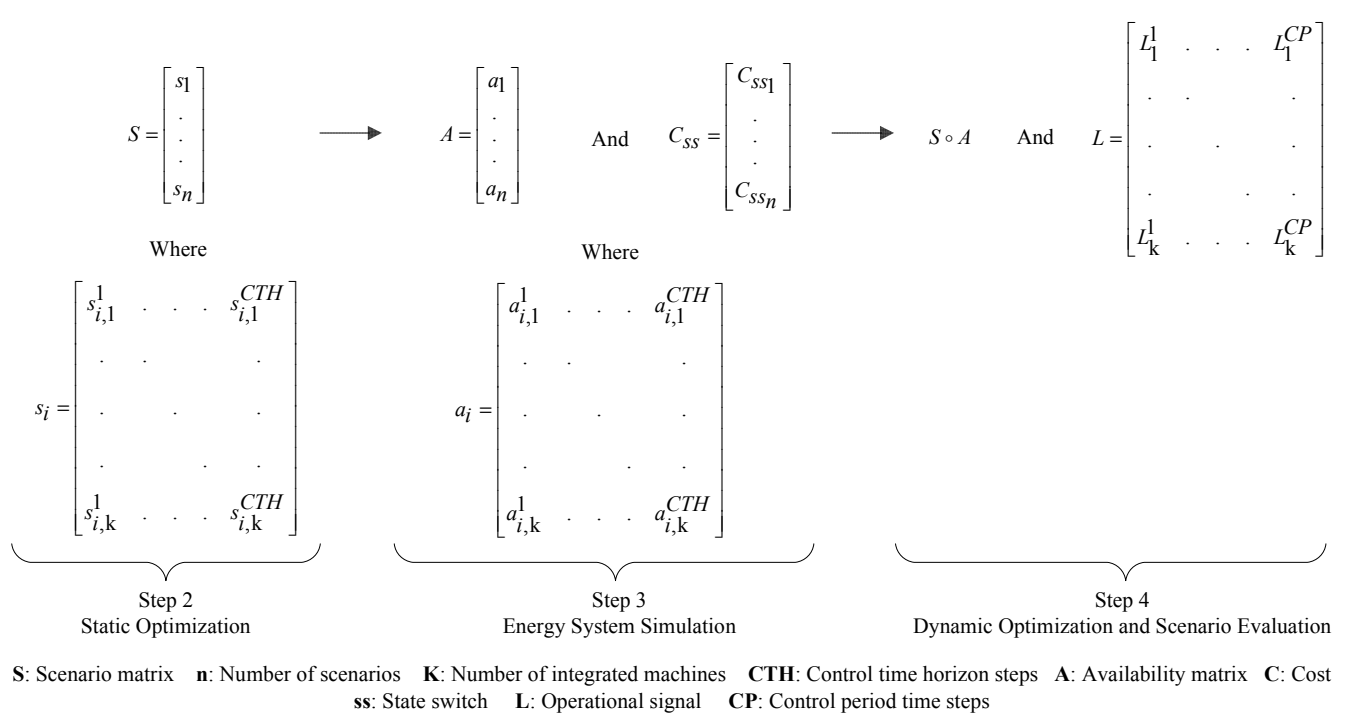

Figure 6. Information flow through RISO methodology steps

\subsubsection{Energy System Simulation}

At this step, based on generated scenario matrix (S), a simulation of energy system operation along the CTH is performed (Figure 4). The latter will generate the associated availability matrix (A) of all the integrated components for individual scenarios and in line with the most recent dynamic operational constraints that contain information from the energy system operational history (Figure 6). Figure 7 illustrates samples of generated A, based on S passed from step two. For example, in case 1 there is no state switch scenario, so the generator will be available along the updated CTH. However in case 2 a switch-off signal at time 17:15 will result in the unavailability of the generator to pass associated cooling down, block and start-up time. Finally, case 3 illustrates the capability of the methodology to take into account the system operation history. In this case the generator is unavailable, as it has been switched off as a result of previous decisions and there is a remaining time to become available, which is encompassed as a dynamic variable.

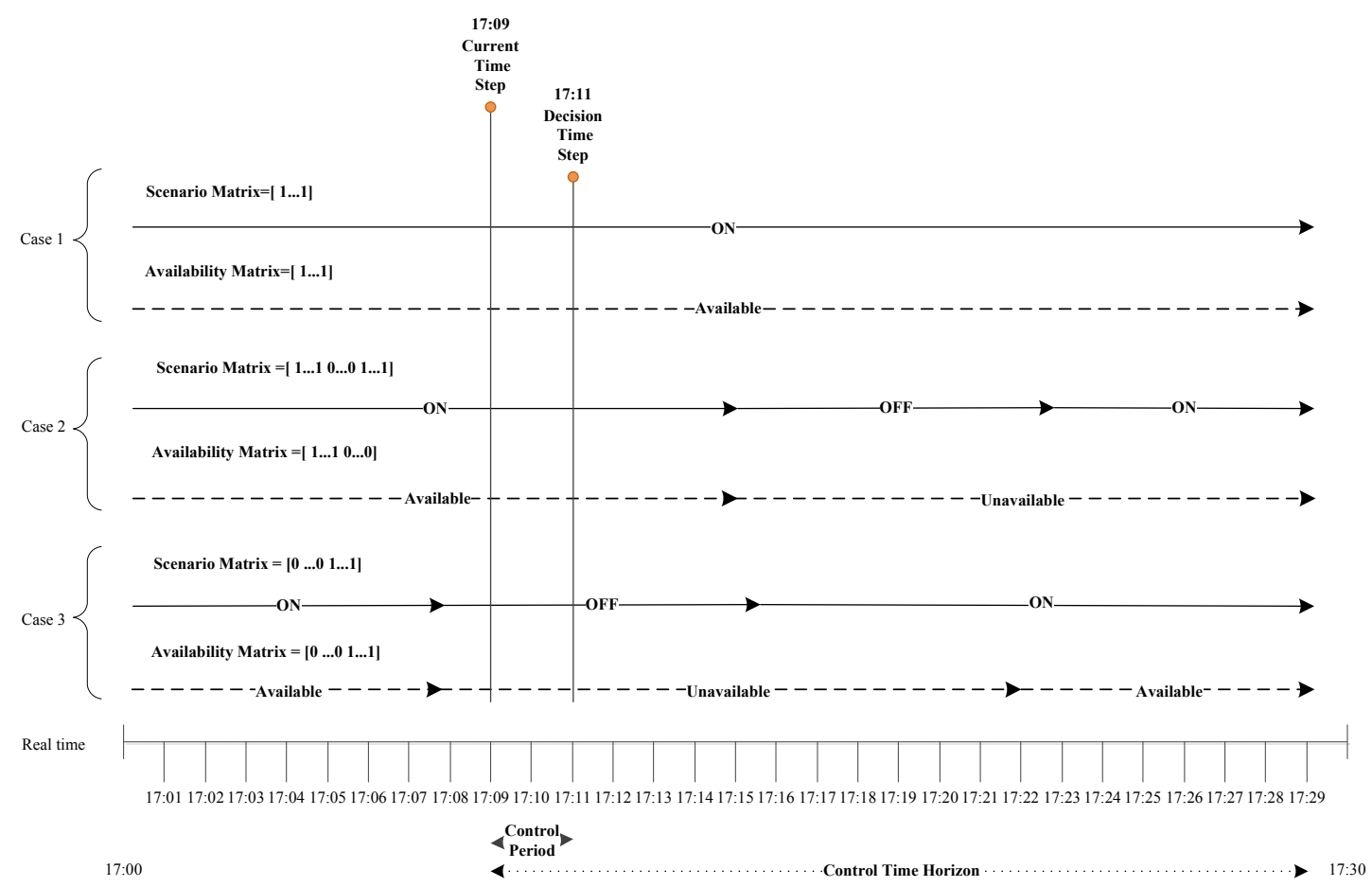

Figure 7. Samples of generated scenarios and their associated availability matrices 
Implementation of the generated $\mathrm{S}$ through the simulation module enables the rapid examination of a large number of scenarios prior to actually implementing a particular decision in the real environment.

In addition, the associated state switch cost for individual scenarios is simulated and assigned to each scenario at this step of the methodology.

\subsubsection{Dynamic Optimization and Scenario Evaluation}

At this step, element-wise multiplication of generated $\mathrm{S}$ and A matrices $(S \circ A)$ will merge the potential scenarios with associated availability of individual generators (Figure 6). Thereafter, based on the generated $S \circ A$ and the state switch cost from the previous step, a linear objective function arises subject to linear equality and inequality constraints. This is solved for individual scenarios by using linear programming (Currie \& Wilson, 2012). As a result, this step covers potential scenarios, the availability of the generators in addition to the interdependency between all individual time steps along the $\mathrm{CTH}$.

Moreover, the optimization module evaluates resulting scenarios and selects the one that satisfies the minimum objective function value. Finally, a matrix (L) is generated, which contains optimal operational signals along the control period (CP) for individual components based on the selected scenario (Figure 6). Consequently although the optimization performed at step two generates optimal operational values for individual time steps, final optimal values are determined by dynamic optimization and scenario evaluation over the CTH at this step. The proposed two-step optimization approach can minimize the search space as a static optimization outcome is used to guide optimum search among all the potential scenarios (Carpaneto et al., 2006).

\subsubsection{State Switch Scheduler}

The simulation module is also used as a tool for optimal scheduling of state switches by backward calculation based on the optimal scenario selected in the previous step and the presence of state switch signals (Figure 4). This considers the sequence of the states and generates appropriate schedules to ensure on-time shutdown or start-up. The latter is also performed with respect to dynamic operational constraints by taking into account the system history. For instance, warm or cold start up conditions can be checked with regards to the last state switch time that will assure reliable start-ups in a dynamic fashion.

\subsubsection{Dynamic Constraint Set}

Optimal operational signals and state switch schedules are implemented during the next control period. Moreover dynamic operational constraints are updated at this stage to be passed to the next run of the methodology (Figure 4).

\subsubsection{Methodology Run Coordinator}

This step is designed to handle the linkage between two consecutive methodology runs. The events at this step are responsible for passing all the generated information to the next run.

\subsubsection{Simulation Run Manager}

Finally, the simulation run manager is integrated to continuously oversee the execution of the methodology and synchronize it with respect to real system conditions (Figure 4). For instance, in case the running time of the methodology takes more than specified constraints, it should generate operational signals in a way that only unit commitment and system reliability is addressed. Moreover external events can be managed at this step along the methodology run.

In summary the proposed methodology decomposes the operation management problem by explicitly modeling, optimizing and scheduling the dispatch problem. This decentralized solution should enable comprehensive modelling, reduce problem complexity and achieve fast problem solving for real-time application without compromising on required reliability.

\subsection{Implementation}

The proposed methodology is capable of being applied iteratively to track a changing optimum as closely as possible in a continuous manner. Figure 8 illustrates the application of the proposed methodology in a closed loop form. 


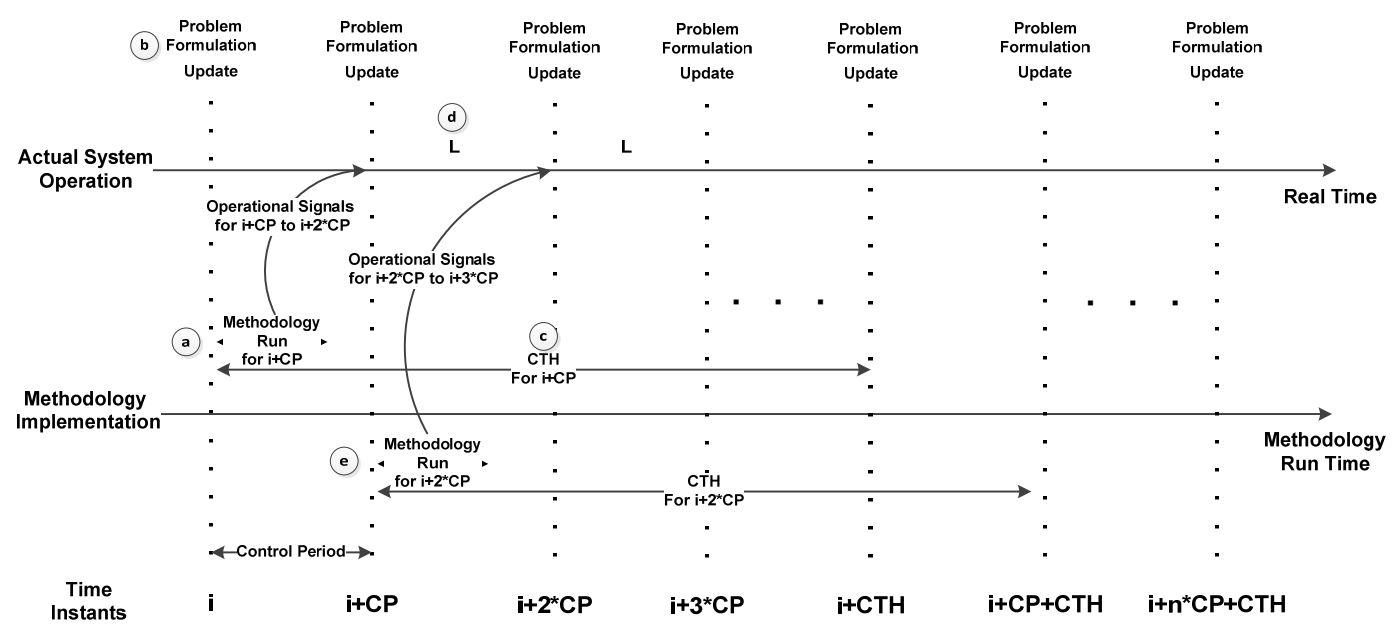

Figure 8. Continuous and iterative application of real-time integrated simulation and optimization methodology

As shown in Figure 8, the core methodology (RISO) is applied at time instant (i) to generate the optimal operational signal matrix (L) for time steps along the control period $\mathrm{i}+\mathrm{CP}$ to $\mathrm{i}+2 * \mathrm{CP}$ (Figure $8-\mathrm{a}$ ). This is done in relation to most updated operational constraints based on the system operation history (Figure 8 -b). On the other hand, the future state of the energy system is taken into account by utilizing the forward-looking capability of the simulation module along the $\mathrm{CTH}$. For instance, operational signals for the upcoming control period (i+CP to $\mathrm{i}+2 * \mathrm{CP}$ ) are generated with regards to the system forecast up to $\mathrm{i}+\mathrm{CTH}$ (Figure $8-\mathrm{c}$ ). Finally, optimal operational signals for time steps between $\mathrm{i}+\mathrm{CP}$ to $\mathrm{i}+2 * \mathrm{CP}(\mathrm{L})$ are implemented (Figure $8-\mathrm{d}$ ) and the next methodology run for control period $\mathrm{i}+2 * \mathrm{CP}$ to $\mathrm{i}+3 * \mathrm{CP}$ is triggered at $\mathrm{i}+\mathrm{CP}$ (Figure $8-\mathrm{e}$ ). As a result, the methodology runs are synchronized with real-time system operation in a continuous manner over the rolling $\mathrm{CTH}$. The next section describes the application of the proposed methodology by using a real industrial case.

\section{Case Study}

The Anylogic simulation package and MATLAB optimization software were used in order to demonstrate the applicability of the proposed methodology. Energy demand data for the studied manufacturing plant was collected and used as the energy demand forecast over the course of two weeks with 15 seconds resolution to achieve a more realistic outcome. The plant's existing on-site energy system consists of two reciprocating engine-based CHP systems, grid interconnection and a boiler. The studied CHP system operates under an electrical load following (ELF) operational strategy in which the engine will be switched on when the demand exceeds $300 \mathrm{~kW}$ over five minutes and switches off when the demand is lower than this threshold for the same duration. Consequently the system functions subject to a fixed operational strategy, which is modelled in the simulation environment. This is used as a baseline scenario to evaluate potential outcomes from the application of the proposed methodology, which aims for economic dispatch of integrated options. The latter is developed based on validated models of the integrated components at the manufacturing plant and previous studies of the authors (Ghadimi et al., 2014a).

\section{Results and Discussion}

In this section, advantages of the proposed methodology are compared with the baseline scenario through analysis of the simulation outcomes. This has been carried out based on two weeks of manufacturing plant operation under the proposed methodology, in which selected time frames are used to illustrate individual advantages compared to the baseline scenario. Finally several objectives are evaluated and compared for the same time frame subject to 15 seconds data resolution, 2 minutes control period and 10 minutes CTH duration.

In this case the following figure illustrates 45 minutes of power demand fluctuations for the studied manufacturing plant. Outcomes from the operation of CHP under the baseline scenario and proposed methodology are also shown. 


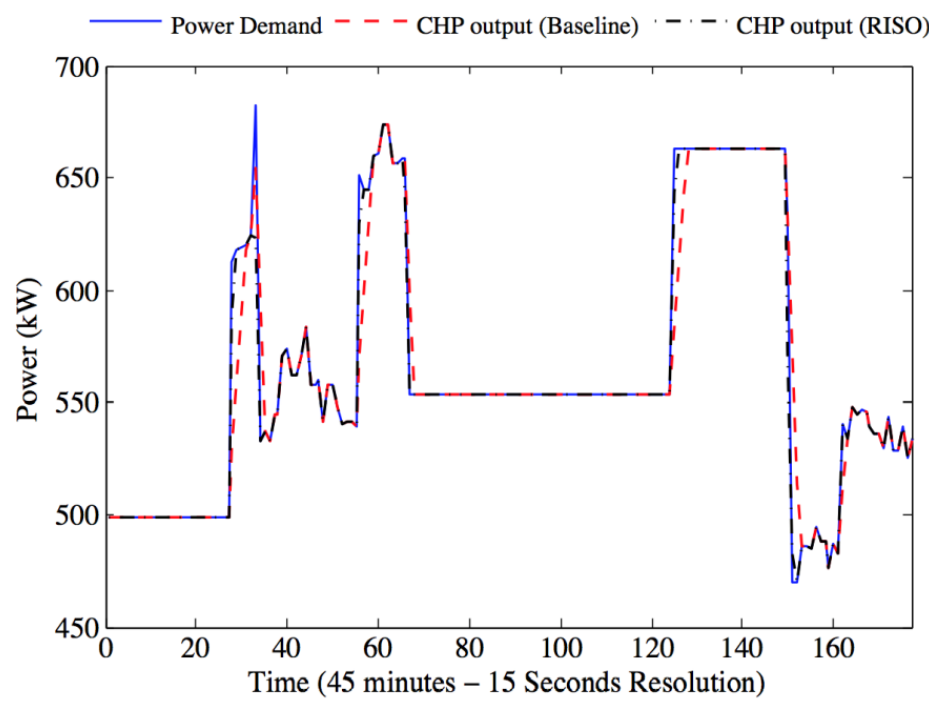

Figure 9. CHP output subject to baseline and RISO operational strategies

It can be seen that the proposed approach has a quicker and more reactive response with regards to power demand fluctuation whereas the baseline scenario shows noticeable latency. Consequently under the proposed methodology and based on the forecasted power demand, integrated generators can response optimally. This is associated with the look-ahead capability of the proposed methodology in capturing system dynamics along the CTH. Several scenarios are defined to illustrate the sensitivity of the proposed methodology in taking into account this capability, in addition to optimal operational signal generation in case there is more flexibility in system operation in the case of storage system presence or opportunity for energy market participation (Figure $10)$.
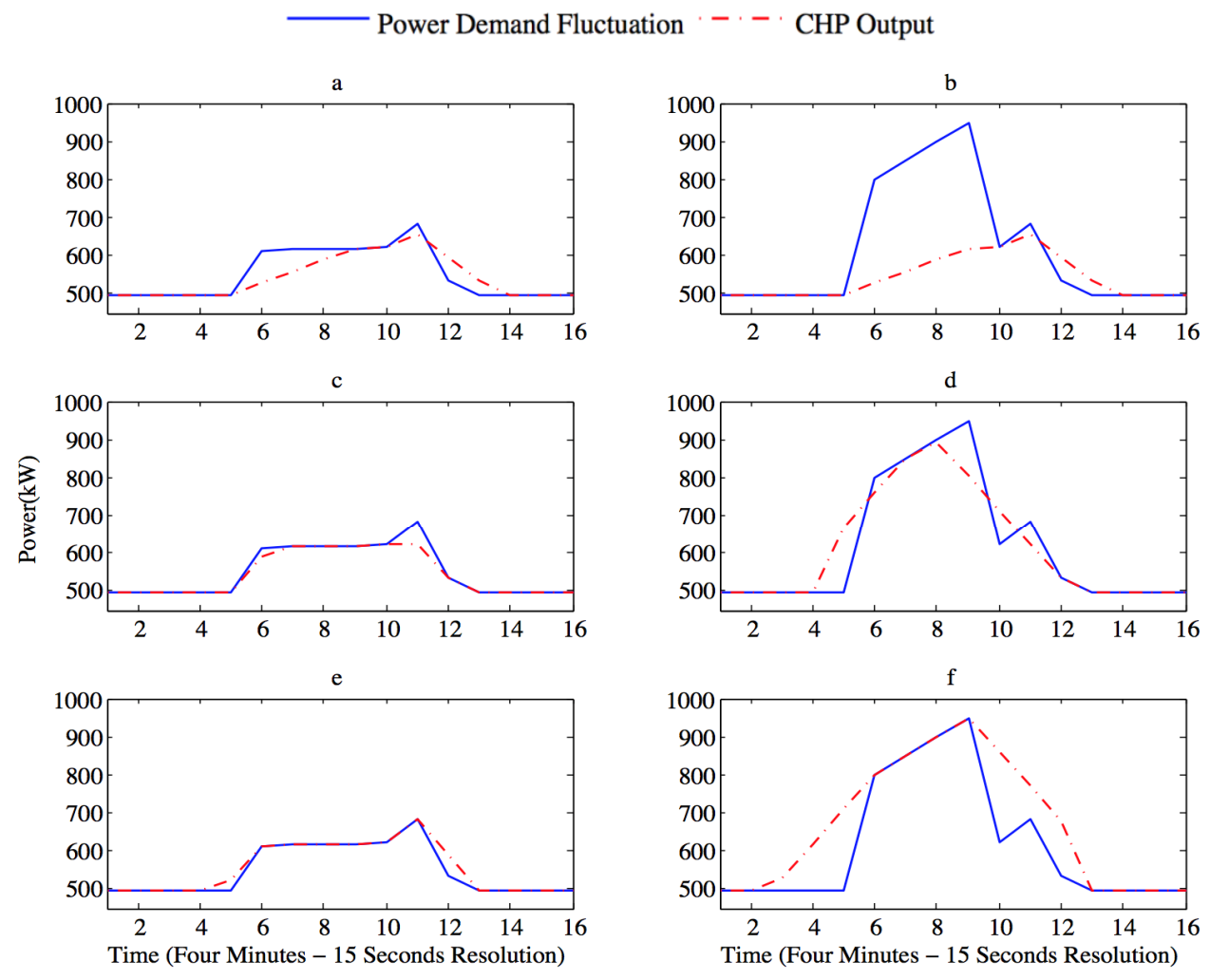

Figure 10. Look-ahead capability and optimal operation signal generation of RISO 
Figure 10 illustrates CHP operation for the studied manufacturing plant under different levels of power demand fluctuation and electricity charges. The first column (Figure $10 \mathrm{a}-\mathrm{c}-\mathrm{e}$ ) illustrates low levels of power demand fluctuation, whereas the second column (Figure $10 \mathrm{~b}-\mathrm{d}-\mathrm{f}$ ) is subject to higher levels. The first row (Figure $10 \mathrm{a}-\mathrm{b}$ ) shows CHP output under the baseline scenario whereas the two other rows are subject to the proposed methodology (Figure $10 \mathrm{c}$-d-e-f). It can be seen that using the proposed methodology (Figure $10 \mathrm{c}-\mathrm{d}$ ), the operational signals are set optimally as compared to the baseline scenario where the CHP output is only reactive to instantaneously applied loads (Figure $10 \mathrm{a}-\mathrm{b}$ ). This is achieved by optimal ramping up and down in response to upcoming fluctuations of the power demand and subject to existing operational constraints. Moreover, the third row (Figure 10 e-f) illustrates the outcomes under a defined scenario, which is subject to high electricity charges. Consequently the operational signals are set to eliminate grid electricity consumption by increasing the CHP output to cover the whole power demand as compared to the scenario in the second row (Figure $10 \mathrm{c}-\mathrm{d}$ ). This is in contrast with the baseline strategy, which has the same CHP output as low electricity charges (Figure 10 a-b) as it doesn't take into account the charges associated with other energy supply options. The latter illustrates the sensitivity of the methodology to manage CHP output as a controllable energy supplier with regards to other potential options. This can add greater value under real-time pricing (RTP) of the energy market, presence of multiple energy suppliers and utilization of energy storage options. Consequently under the proposed methodology, generators can optimally manage their ramp up/down to achieve defined objectives subject to existing constraints.

Furthermore, an advantage of the proposed methodology over CHP state-switch control is illustrated in the following figure. In this case, subject to the baseline scenario, the engine's state switch (on/off) is triggered solely based on the level and duration of power demands (ELF operational strategy), however it is not an economical approach (Figure 11).

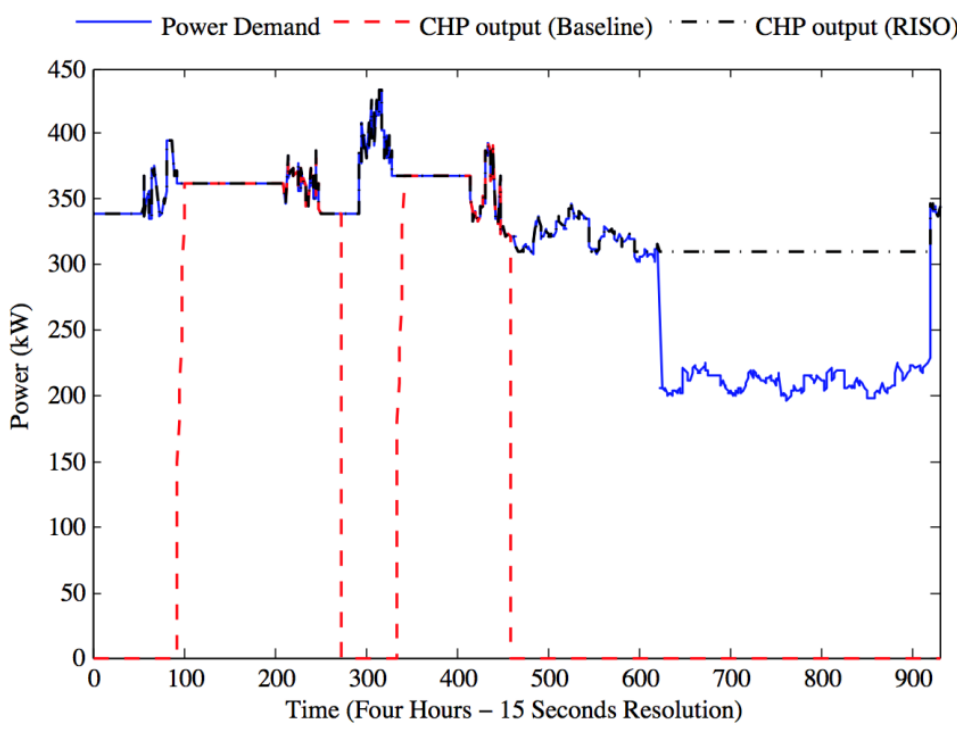

Figure 11. CHP state switch subject to baseline and RISO operational strategy

In contrast, subject to the proposed methodology, the CHP operates so as to avoid extra grid or associated state switch charges. This is achieved by taking into account the power demand forecast along the CTH in addition to charges associated with other potential energy supply options. Consequently this evaluates if state-switch satisfies the minimum objective values along the CTH or not.

Finally, the methodology is capable of efficiently scheduling the engine's start-ups and shutdowns. This is illustrated based on the defined scenario in the following figure. In this case, the proposed methodology foresees the power demand increase and receives a state-switch signal, which triggers and schedules the engine's start-up at time step 35. This is in contrast with the baseline scenario with latency, in which the system is dependent on power demand monitoring for a specific duration to be triggered at time step 100. 


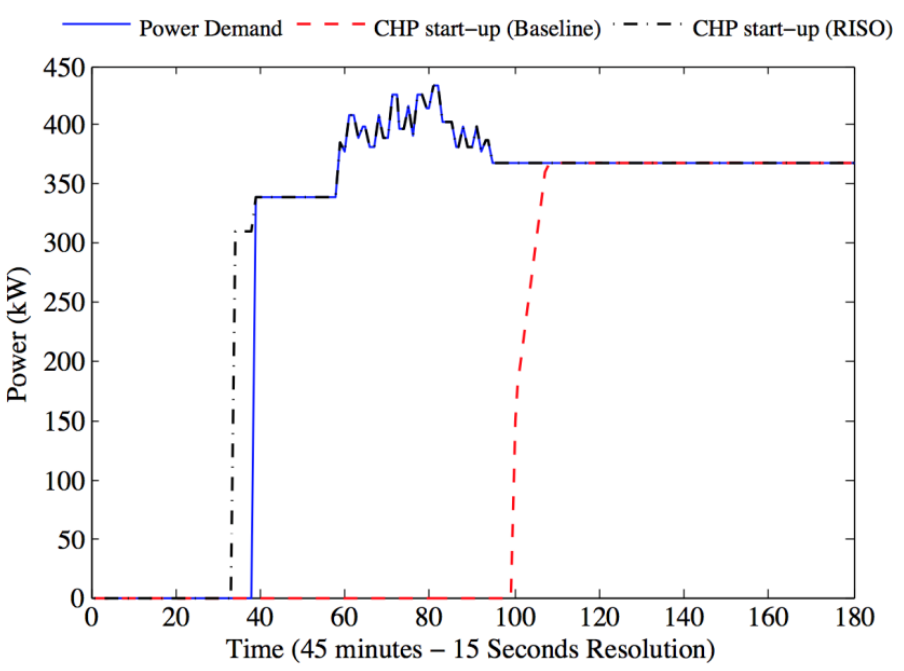

Figure 12. CHP start-up subject to baseline and RISO operational strategy

The CHP operation subject to RISO and baseline scenarios is illustrated over one week in figure 13 in order to evaluate the overall performance of the on-site energy system. In addition, the equivalent environmental impact $(\mathrm{CO} 2 \mathrm{e})$ and primary energy consumption $(\mathrm{kWh})$ are calculated to give more insight into different aspects of the proposed operational strategy.
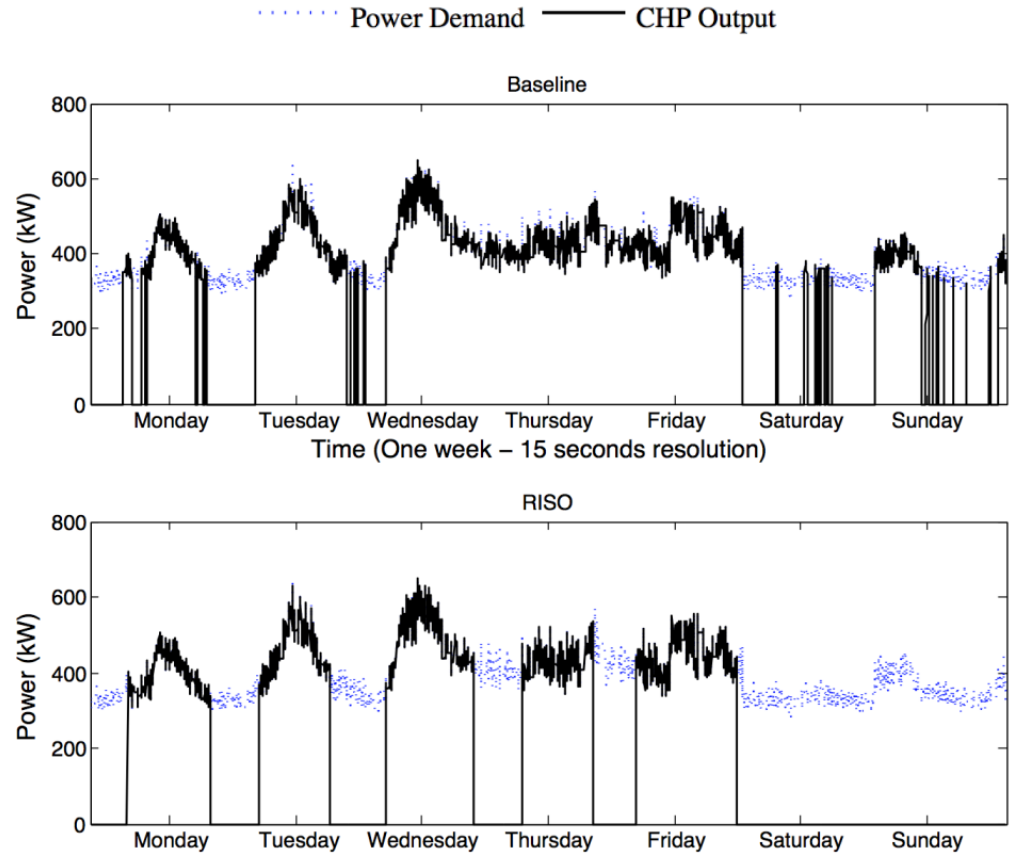

Figure 13. CHP output subject to baseline and RISO operational strategies over one week

It can be observed that purchased electricity charges can play a vital role in the optimal economic operation of the on-site energy system. In this case, the engine switches to off mode under RISO application to prevent additional operation cost even though during off-peak charge hours the power demand is high.

The following plot (Figure 14) compares selected economic, environmental and energy consumption indicators over a week for one engine. In this case, under RISO, the economical dispatch results in lower energy charges (Figure 14-a). However, the environmental impact (Figure 14-h) and primary energy consumption (Figure 14-g) are increased. The latter is due to a higher share of electricity supply through grid interconnection, subject to RISO (Figure 14-f), which is mainly generated by coal-fired power plants with high levels of environmental 
impact and low levels of operational efficiency ( $\sim 30 \%)$ compared to CHP options.

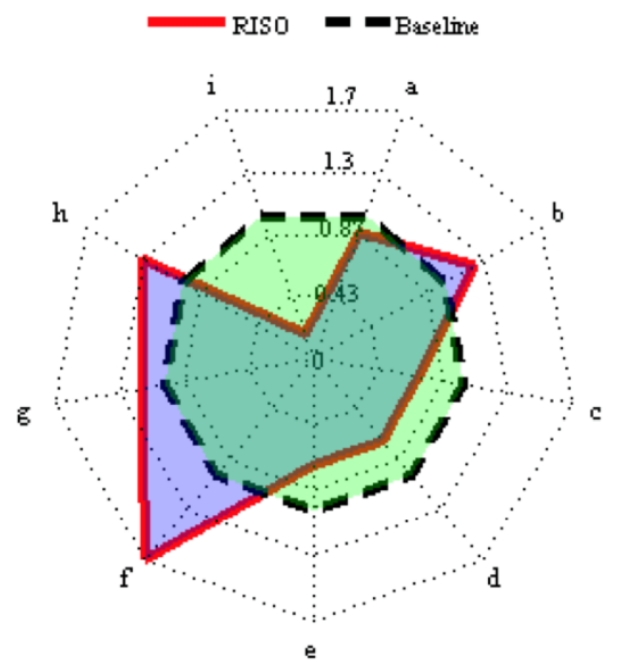

\begin{tabular}{lrr}
\hline \multicolumn{1}{c}{ Objectives } & Baseline & RISO \\
\hline a- Total Manufacturing Plant Energy Charges (\$) & 3241 & 2853 \\
b- Total Charge of Purchased Electricity (\$) & 1123 & 1374 \\
c- Total Charge of CHP Operation (\$) & 2117 & 1479 \\
d- Total Generated Energy by CHP $(\mathrm{kWh})$ & 46808 & 32928 \\
e- Total Fuel Consumption of CHP (kWh) & 70037 & 48923 \\
f- Total Purchased Electricity (kWh) & 18878 & 32724 \\
g- Total Primary Energy Consumption (kWh) & 125561145171 \\
h- Total Environmental Impact - CO2e (kg) & 31213 & 40719 \\
i- Number of State Switches & 58 & 10 \\
\hline
\end{tabular}

Figure 14. Economic and environmental objective evaluation subject to baseline and RISO operational strategies

Furthermore, the proposed approach not only satisfies objectives, it also minimises the number of state switches (Figure 14-i) and this can have positive business impacts such as maintenance cost reduction.

Finally it should be noted that selection of the parameters that relate to methodology implementation could be critical. In this case, resolution of input data, in addition to the duration of the control period and the CTH should be identified accordingly. These should be analysed and set for individual systems, as each have their own specific demand and supply characteristics. For instance, the duration of the CTH should be matched with the longest start-up or shutdown time to guarantee optimal state switch triggering. On the other hand, resolution of the incorporated data will define precision with which decisions may be made. Finally a trade-off to select the best values is required as all these parameters can impact the time that is required to run the methodology. This should guarantee continuous application where execution time of the methodology should be lower than the control period.

In our case study, computational statistics for individual methodology runs were as follows. Static optimization with five decision variables, three binary variables and eighteen constraints for 40 time steps took on average 0.0016 seconds. Dynamic optimization with eighty decision variables, two hundred and thirty eight constraints took 0.0007 seconds to reach an optimal result. In total, each run for an individual iteration took an average of thirteen seconds and this will allow real-time implementation. These results have been achieved on an Intel ${ }^{\circledR}$ Core ${ }^{\mathrm{TM}}$ i5-2500 CPU @ 3.3GHZ system with $4096 \mathrm{Mb}$ memory.

\section{Conclusion}

Whereas efficient use of on-site energy generation options is currently limited to optimal sizing and static operational strategy selection, this paper proposed a methodology to facilitate autonomous operational management of the controllable options in a dynamic fashion. The developed real-time integrated simulation and optimization methodology ensures a reliable and intelligent control strategy, which can be applied iteratively in dynamic operating environments. The latter outcome is accomplished through the integration of simulation and optimization modules to facilitate coverage of concurrencies, hierarchical arrangements and interdependencies between components.

Using this approach, reactive models of potential components, which can take into account operational states and associated transitions, have been developed. These models also overcome the shortcomings of existing approaches in providing for real-time applicability and communication requirements. Consequently the models represent component behavior in short time intervals and can also be linked to the dynamic operating environment in order to receive feedback and to react to external events. This is in contrast with time driven systems. Additionally, the proposed approach considers both system history and a forecast of its operation along the control time horizon. The aforementioned advantages would guarantee reliability of the models in representing both the component and system levels of operation. In addition, optimal operational signals are generated via an optimization module that generates potential scenarios and selects the optimal solution to 
guarantee minimum dispatch costs. Finally, optimal state switch schedules are developed accordingly.

Consequently the problem is decomposed into sub-problems at different levels and modules to avoid the complexity associated with pure mathematical modelling approaches. The latter minimises modelling overload and makes the problem computationally inexpensive to be executed iteratively in real-time without compromising model reliability as compared to existing approaches.

Finally, this study illustrates the potential outcomes of the proposed methodology in the presence of dynamic operational constraints when compared to a baseline scenario in which the CHP system is operated under a static operational strategy. Consequently this study integrates the essential requirements for real-time operational management of on-site energy systems. This will result in improved stability in the presence of external events and operating environment dynamics, as well as scalability and complex problem handling that should lead to optimal and on-line decision-making.

Further research will be conducted in circumstances where the energy system becomes more complex in the presence of multiple generating options, such as renewable energy with intermittent output and energy storage technologies. Moreover, real-time data should be integrated into the problem formulation.

\section{Acknowledgments}

This project is funded by the Australian Research Council in collaboration with Baxter Healthcare Australia.

\section{References}

Andreassi, L., Ciminelli, M. V., Feola, M., \& Ubertini, S. (2009). Innovative method for energy management: Modelling and optimal operation of energy systems. Energy and Buildings, 41(4), 436-444. http://dx.doi.org/10.1016/j.enbuild.2008.11.010

Arroyo, J. M., \& Conejo, A. J. (2004). Modeling of start-up and shut-down power trajectories of thermal units. IEEE Transactions on Power Systems, 19(3), 1562-1568. http://dx.doi.org/10.1109/TPWRS.2004.831654

Baños, R., Manzano-Agugliaro, F., Montoya, F. G., Gil, C., Alcayde, A., \& Gómez, J. (2011). Optimization methods applied to renewable and sustainable energy: A review. Renewable and Sustainable Energy Reviews, 15(4), 1753-1766. http://dx.doi.org/10.1016/j.rser.2010.12.008

Bausa, J., \& Tsatsaronis, G. (1999). Dynamic Optimization of Startup and Load-Increasing Processes in Power Plants-Part I: Method. Journal of Engineering for Gas Turbines and Power, 123(1), 246-250. http://dx.doi.org/10.1115/1.1286728

Bonvin, D., Srinivasan, B., \& Ruppen, D. (2002). Dynamic optimization in the batch chemical industry. In AIChE Symposium Series (pp. 255-273). New York; American Institute of Chemical Engineers; 1998. Retrieved from http://infoscience.epfl.ch/record/28363/files/fulltext.pdf

Carpaneto, E., Cavallero, C., Freschi, F., \& Repetto, M. (2006). Immune Procedure for Optimal Scheduling of Complex Energy Systems. In H. Bersini \& J. Carneiro (Eds.), Artificial Immune Systems (pp. 309-320). Springer Berlin Heidelberg. http://dx.doi.org/10.1007/11823940_24

Carrión, M., \& Arroyo, J. M. (2006). A computationally efficient mixed-integer linear formulation for the thermal unit commitment problem. IEEE Transactions on Power Systems, 21(3), 1371-1378. http://dx.doi.org/10.1109/TPWRS.2006.876672

Carvalho, M., Romero, A., Shields, G., \& Millar, D. L. (2014). Optimal synthesis of energy supply systems for remote open pit mines. Applied Thermal Engineering, 64(1-2), 315-330. http://dx.doi.org/10.1016/j.applthermaleng.2013.12.040

Chang, G. W., Tsai, Y. D., Lai, C. Y., \& Chung, J. S. (2004). A practical mixed integer linear programming based approach for unit commitment. In IEEE Power Engineering Society General Meeting, 2004 (Vol. 1, pp. 221-225). http://dx.doi.org/10.1109/PES.2004.1372789

Cho, H., Luck, R., \& Chamra, L. M. (2010). Supervisory Feed-Forward Control for Real-Time Topping Cycle CHP Operation. Journal of Energy Resources Technology, 132(1), 012401-12. http://dx.doi.org/10.1115/1.4000920

Colson, C. M., Nehrir, M. H., \& Pourmousavi, S. A. (2010). Towards real-time microgrid power management using computational intelligence methods. In 2010 IEEE Power and Energy Society General Meeting (pp. 1-8). IEEE. http://dx.doi.org/10.1109/PES.2010.5588053

Currie, J., \& Wilson, D. I. (2012). OPTI: lowering the barrier between open source optimizers and the industrial 
MATLAB user. Foundations of Computer-Aided Process Operations, Savannah, Georgia, USA, 8-11.

Dillon, T. S., Edwin, K. W., Kochs, H. D., \& Taud, R. J. (1978). Integer Programming Approach to the Problem of Optimal Unit Commitment with Probabilistic Reserve Determination. IEEE Transactions on Power Apparatus and Systems, PAS-97(6), 2154-2166. http://dx.doi.org/10.1109/TPAS.1978.354719

Dimeas, A. L., \& Hatziargyriou, N. D. (2005). Operation of a Multiagent System for Microgrid Control. IEEE Transactions on Power Systems, 20(3), 1447- 1455. http://dx.doi.org/10.1109/TPWRS.2005.852060

Douglass, B. P. (2002). Real-Time UML. In W. Damm \& E.-R. Olderog (Eds.), Formal Techniques in Real-Time and Fault-Tolerant Systems (pp. 53-70). Springer Berlin Heidelberg. http://dx.doi.org/10.1007/3-540-45739-9_4

Firestone, R. M., \& Berkeley, U. of C. (2007). Optimal real-time dispatch for integrated energy systems. University of California, Berkeley.

Flores, R. J., Shaffer, B. P., \& Brouwer, J. (2014). Dynamic distributed generation dispatch strategy for lowering the cost of building energy. Applied Energy, 123, 196-208. http://dx.doi.org/10.1016/j.apenergy.2014.02.028

Gamou, S., Yokoyama, R., \& Ito, K. (2002). Optimal unit sizing of cogeneration systems in consideration of uncertain energy demands as continuous random variables. Energy Conversion and Management, 43(9-12), 1349-1361. http://dx.doi.org/10.1016/S0196-8904(02)00020-1

Ghadimi, P., Kara, S., \& Kornfeld, B. (2013). Advanced On-Site Energy Generation towards Sustainable Manufacturing. In A. Y. C. Nee, B. Song, \& S. K. Ong (Eds.), Re-engineering Manufacturing for Sustainability (pp. 153-158). Springer Singapore. http://dx.doi.org/10.1007/978-981-4451-48-2_25

Ghadimi, P., Kara, S., \& Kornfeld, B. (2014a). Reactive modelling of on-site energy system components for real-time application. In 2014 IEEE International Conference on Intelligent Energy and Power Systems (IEPS) (pp. 219-224). http://dx.doi.org/10.1109/IEPS.2014.6874183

Ghadimi, P., Kara, S., \& Kornfeld, B. (2014b). The optimal selection of on-site CHP systems through integrated sizing and operational strategy. Applied Energy, 126, 38-46. http://dx.doi.org/10.1016/j.apenergy.2014.03.085

Herrmann, C., Thiede, S., Kara, S., \& Hesselbach, J. (n.d.). Energy oriented simulation of manufacturing systems - Concept and application. CIRP Annals - Manufacturing Technology, In Press, Corrected Proof. http://dx.doi.org/10.1016/j.cirp.2011.03.127

Hiremath, R. B., Shikha, S., \& Ravindranath, N. H. (2007). Decentralized energy planning; modeling and application-a review. Renewable and Sustainable Energy Reviews, 11(5), 729-752. http://dx.doi.org/10.1016/j.rser.2005.07.005

Hueffed, A. K., \& Mago, P. J. (2010). Influence of prime mover size and operational strategy on the performance of combined cooling, heating, and power systems under different cost structures. Proceedings of the Institution of Mechanical Engineers, Part A: Journal of Power and Energy, 224(5), 591-605. http://dx.doi.org/10.1243/09576509JPE922

Kim, H. M., Kinoshita, T., \& Shin, M. C. (2010). A Multiagent System for Autonomous Operation of Islanded Microgrids Based on a Power Market Environment. Energies, 3(12), 1972-1990. http://dx.doi.org/10.3390/en3121972

Kim, H. M., Lim, Y., \& Kinoshita, T. (2012). An Intelligent Multiagent System for Autonomous Microgrid Operation. Energies, 5(9), 3347-3362. http://dx.doi.org/10.3390/en5093347

Kraning, M., Chu, E., Lavaei, J., \& Boyd, S. (2012). Message Passing for Dynamic Network Energy Management. arXiv:1204.1106 [cs, Math]. Retrieved from http://arxiv.org/abs/1204.1106

Lee, F. N., Lemonidis, L., \& Liu, K. (1994). Price-based ramp-rate model for dynamic dispatch and unit commitment. IEEE Transactions on Power Systems, 9(3), 1233-1242. http://dx.doi.org/10.1109/59.336075

Liu, M., Shi, Y., \& Fang, F. (2013). Optimal power flow and PGU capacity of CCHP systems using a matrix modeling approach. Applied Energy, 102, 794-802. http://dx.doi.org/10.1016/j.apenergy.2012.08.041

Logenthiran, T., Srinivasan, D., Khambadkone, A., \& Aung, H. N. (2012). Multiagent System for Real-Time Operation of a Microgrid in Real-Time Digital Simulator. IEEE Transactions on Smart Grid, 3(2), 925-933. http://dx.doi.org/10.1109/TSG.2012.2189028 
Lozano, M. A., Carvalho, M., \& Serra, L. M. (2011). Allocation of economic costs in trigeneration systems at variable load conditions. Energy and Buildings, 43(10), 2869-2881. http://dx.doi.org/10.1016/j.enbuild.2011.07.002

Marshman, D. J., Chmelyk, T., Sidhu, M. S., Gopaluni, R. B., \& Dumont, G. A. (2010). Energy optimization in a pulp and paper mill cogeneration facility. Applied Energy, 87(11), 3514-3525. http://dx.doi.org/10.1016/j.apenergy.2010.04.023

Mayhorn, E., Kalsi, K., Elizondo, M., Zhang, W., Lu, S., Samaan, N., \& Butler-Purry, K. (2012). Optimal control of distributed energy resources using model predictive control. In 2012 IEEE Power and Energy Society General Meeting (pp. 1-8). http://dx.doi.org/10.1109/PESGM.2012.6345596

Meckler, M., \& Hyman, L. (2009). Sustainable On-Site CHP Systems: Design, Construction, and Operations: Design, Construction, and Operations. McGraw Hill Professional.

Meiqin, M., Wei, D., \& Chang, L. (2011). Multi-agent based simulation for Microgrid energy management. In 2011 IEEE 8th International Conference on Power Electronics and ECCE Asia (ICPE ECCE) (pp. 1219-1223). http://dx.doi.org/10.1109/ICPE.2011.5944680

Mitra, S., Grossmann, I. E., Pinto, J. M., \& Arora, N. (2012). Optimal production planning under time-sensitive electricity prices for continuous power-intensive processes. Computers \& Chemical Engineering, 38, 171-184. http://dx.doi.org/10.1016/j.compchemeng.2011.09.019

Mitra, S., Sun, L., \& Grossmann, I. E. (2013). Optimal scheduling of industrial combined heat and power plants under time-sensitive electricity prices. Energy, 54, 194-211. http://dx.doi.org/10.1016/j.energy.2013.02.030

Müller, E., Stock, T., \& Schillig, R. (2014). Energy Value-Stream Mapping - A Method to Optimize Value-Streams in Respect of Time and Energy Consumption. In M. F. Zaeh (Ed.), Enabling Manufacturing Competitiveness and Economic Sustainability (pp. 285-290). Springer International Publishing. http://dx.doi.org/10.1007/978-3-319-02054-9_48

Oh, S. D., Lee, H. J., Jung, J. Y., \& Kwak, H. Y. (2007). Optimal planning and economic evaluation of cogeneration system. Energy, 32(5), 760-771. http://dx.doi.org/10.1016/j.energy.2006.05.007

Rong, A., Hakonen, H., \& Lahdelma, R. (2009). A dynamic regrouping based sequential dynamic programming algorithm for unit commitment of combined heat and power systems. Energy Conversion and Management, 50(4), 1108-1115. http://dx.doi.org/10.1016/j.enconman.2008.12.003

Salgado, F., \& Pedrero, P. (2008). Short-term operation planning on cogeneration systems: A survey. Electric Power Systems Research, 78(5), 835-848. http://dx.doi.org/10.1016/j.epsr.2007.06.001

Smitha, S. D., \& Chacko, F. M. (2013). Intelligent energy management in smart and sustainable buildings with multi-agent control system. In 2013 International Multi-Conference on Automation, Computing, Communication, Control and Compressed Sensing (iMac4s) (pp. 190-195). http://dx.doi.org/10.1109/iMac4s.2013.6526406

Turner, W. C. (2004). Energy Management Handbook. Marcel Dekker.

Vallianou, V. A., \& Frangopoulos, C. A. (2012). Dynamic Operation Optimization of a Trigeneration System. International Journal of Thermodynamics, 15(4), 239-247. http://dx.doi.org/10.5541/ijot.382

Wille-Haussmann, B., Erge, T., \& Wittwer, C. (2010). Decentralised optimisation of cogeneration in virtual power plants. Solar Energy, 84(4), 604-611. http://dx.doi.org/10.1016/j.solener.2009.10.009

Xie, L., \& Ilić, M. D. (2009). Model predictive economic/environmental dispatch of power systems with intermittent resources. In IEEE Power Energy Society General Meeting, 2009. PES '09 (pp. 1-6). http://dx.doi.org/10.1109/PES.2009.5275940

Yun, K., Cho, H., Luck, R., \& Mago, P. J. (2011). Real-time combined heat and power operational strategy using a hierarchical optimization algorithm. Proceedings of the Institution of Mechanical Engineers, Part A: Journal of Power and Energy, 225(4), 403-412. http://dx.doi.org/10.1177/2041296710394287

\section{Copyrights}

Copyright for this article is retained by the author(s), with first publication rights granted to the journal.

This is an open-access article distributed under the terms and conditions of the Creative Commons Attribution license (http://creativecommons.org/licenses/by/3.0/). 